\title{
Why and when PCI, why and when thrombolysis?
}

\section{PCI}

\author{
Maddalena Lettino
}

Received: 11 November 2008/ Accepted: 18 November 2008/Published online: 13 December 2008 (c) SIMI 2008

Reperfusion therapy has a central role in the treatment of patients with ST-elevation myocardial infarction (STEMI) because, if timely performed, it contributes to reduce infarct size and to preserve left ventricular function. In all STEMI patients with symptom onset within $12 \mathrm{~h}$, restoration of normal coronary flow of the infarct-related artery (IRA) as soon as possible is a class I recommendation according to the available international guidelines [1, 11]. Compared with no reperfusion, reopening of IRA reduces early and late mortality.

Mechanical percutaneous revascularization in the emergent context of myocardial infarction (MI) is named primary PCI and might be superior to treatment with fibrinolytic agents, particularly in patients at high risk for major cardiac adverse events, with prior MI or who present to the hospital more than $4 \mathrm{~h}$ after symptom onset [2].

Several randomized trials have evaluated the benefits of primary angioplasty as compared to fibrinolysis.

In 2003, Keeley et al. [3] identified 23 randomized trials that together randomly assigned 7,739 thrombolytic-eligible patients to primary PCI $(n=3,872)$ or to fibrinolytic therapy ( $n=3,867)$; in the majority of patients, the fibrinolytic drug was a fibrin-specific one, streptokinase being administered only in 8 trials $(n=1,837)$. Considering mechanical reperfusion, stents were used in 12 trials and GPIIbIIIa receptor inhibitors were administered in eight trials of primary PCI. Evaluating both short-term and longterm clinical outcomes, primary PCI was better than thrombolysis at reducing overall death, MI, stroke and the combined endpoint of the three of them; the benefit

M. Lettino $(\bowtie)$

Fondazione IRCCS Policlinico S. Matteo, piazza Golgi 2,

27100 Pavia, Italy

e-mail:m.lettino@smatteo.pv.it reported with primary PCI was still present during longterm follow up and independent of both the types of thrombolytic agent used, and whether or not the patients were transferred from a hospital to another for primary PCI. The result of the meta-analysis is in accordance with previously published data concerning more than 3,700 patients, randomized in some clinical trials to on-site thrombolysis versus primary PCI, who requested a transfer to another hospital with a transfer time shorter than $3 \mathrm{~h}$. Patients treated with primary PCI showed a significant reduction of the primary combined endpoint of death, reinfarction and stroke, with a trend toward reduction of all-cause mortality with transfer for PCI [4].

Although the benefit of primary PCI in randomized clinical trials could be partially attributed to the selection of low risk patients, several prospective registries have confirmed the better survival in real world patients treated with angioplasty. Stenestrand et al. [5] evaluated the outcome of different reperfusion strategies by a prospective observational cohort study of 26,205 consecutive STEMI patients in the Register of Information and Knowledge about Swedish Heart Intensive Care Admission (RIKSHIA), all of whom received reperfusion therapy within $15 \mathrm{~h}$ of symptom onset. More than 19,000 patients received fibrinolytic therapy: of them about 16,000 as in-hospital treatment and 3,078 as pre-hospital treatment. The remaining 7,084 underwent primary PCI. Median delay time from onset of symptoms to the initiation of reperfusion was $210 \mathrm{~min}$ for patients treated by PCI (symptom onset to balloon inflation), $167 \mathrm{~min}$ for in-hospital fibrinolysis (symptom onset to needle insertion) and 120 for pre-hospital fibrinolysis. Both patients who underwent primary PCI and patients who received pre-hospital fibrinolysis were younger and had fewer comorbidities as compared to patients treated by in-hospital fibrinolysis. 
After adjusting for younger age and fewer comorbidities, primary PCI was associated with a lower mortality at 30 days than in-hospital fibrinolysis and correlated also with lower mortality at 30 days as compared to pre-hospital thrombolysis. This was mainly observed in patients who arrive more than $2 \mathrm{~h}$ after symptom onset. Primary PCI was also associated with a shorter hospital stay and a lower incidence of reinfarction as compared to fibrinolytic treatment, administered both in hospital and in the prehospital setting.

Primary PCI shows some potential advantages, compared to fibrinolysis, supporting its role as first choice treatment for patients who arrive at a hospital with a welltrained PCI capability and are potentially candidates for either reperfusion strategy. In fact, PCI is suitable for more than $90 \%$ of patients (thrombolysis can be applied only to $60-80 \%$ of the presenting population), establishes initial TIMI flow grade 3 in $70-90 \%$ of cases and nearly eliminates the risk of intracranial hemorrhage [6]. Finally, coronary angioplasty carries no risk of inappropriate treatment.

\section{What about time?}

It is well known that fibrinolytic therapy is less efficacious in restoring blood flow in the IRA when the interval between the onset of symptom and treatment increases and this is more evident beyond a cut-off of $3 \mathrm{~h}$. Pre-hospital fibrinolysis reduces the delay from the first medical contact to needle insertion time and in this way it reduces mortality [7]. In contrast, the successful opening of the infarct related artery in patients undergoing primary PCI is less dependent on symptom duration, even though it has been reported that time is associated with outcome in high-risk patients. De Luca et al. have evaluated the relationship between time to treatment and mortality as a continuous function, in a cohort of 1,791 patients with STEMI who underwent mechanical revascularization, estimating the risk of mortality for each 30-min delay. The authors conclude that primary PCI affects 1-year mortality for every minute of delay from the onset of symptom to balloon inflation, even when results are adjusted for age, gender, diabetes and previous revascularization [8]. This is easily comprehensible because mechanical reperfusion might result in a good coronary flow, but the ischemic time of the myocardium might have been long enough to produce a large necrotic area, which in turn affects prognosis and longterm mortality. Based on the scientific evidences available, both the Task Force on the Management of Acute Myocardial Infarction of the European Society of Cardiology and the ACC/AHA Task Force on practice guidelines recommend a target medical contact- or door-to-balloon time of less than $90 \mathrm{~min}$ in all STEMI patients addressed for primary PCI.

\section{Do we have to address all STEMI patients to primary PCI?}

PCI appears to have its greatest mortality benefits in highrisk patients, including patients with large anterior MI or hemodynamic impairment in Killip Class III-IV. This is demonstrated in the SHOCK trial, where patients in cardiogenic shock have an absolute 9\% reduction in 30-day mortality with coronary revascularization instead of immediate medical stabilization [9]. Primary PCI is also mandatory in patients at high risk for bleeding complications (mainly at risk for intracranial hemorrhage or severe bleeding) in whom fibrinolytic therapy is contraindicated.

In hospitals with a PCI capability, mechanical revascularization should be timely performed in all patients, aiming to maintain the door-to-balloon time below $90 \mathrm{~min}$, independent of the risk profile of the patient.

In all other situations, and particularly in hospitals, where a catheterization laboratory is not available, the choice between medical or mechanical reperfusion is based upon the time from symptom onset of the ischemic process and the time of delay between a prompt fibrinolytic treatment and a deferred PCI. Using data from 21 trials enrolling patients with STEMI, Nallamothu and Bates evaluated both the 4 to 6 -week incidence of death, reinfarction and stroke in patients who received thrombolysis or primary PCI and the PCI related time delay, defined as the mean (or median) difference between time-to-needle insertion in fibrinolytic therapy and time-to-balloon inflation in PCI. They found that as PCI related time delay increased, absolute mortality reduction at 4-6 weeks favouring primary PCI over fibrinolytic treatment decreases, with apparent equivalence after a PCI-related time delay of more than an hour [10]. This is the reason why in patients with a symptom duration within $3 \mathrm{~h}$, the AHA/ ACC guidelines on STEMI state that primary PCI should be preferred to fibrinolytic treatment, whether or not the hospital where the patient arrives has a PCI capability, if the expected door-to-balloon inflation time minus the doorto-needle insertion time is less than $1 \mathrm{~h}$ (class I, level of evidence B) [11].

Finally, availability of invasive facilities is an important determinant of feasibility of PCI: the catheterization laboratory team should be well trained, and should perform a high volume of procedures per year; moreover, organization of ambulance systems and an adequate pre-hospital management are also required to ultimately provide a successful reperfusion therapy in the majority of patient candidates to primary PCI [12]. 


\section{Conclusion}

Primary PCI has many advantages over fibrinolytic treatment, and should be specifically recommended in all patients at high risk for death, reinfarction or hemorrhagic stroke. As with fibrinolysis, benefit from primary PCI is also time related, even though after $3 \mathrm{~h}$ of symptom onset there are still good chances to reopen the infarct-related coronary artery and to positively influence the outcome of the patient. In any case, a good interaction between ambulance system and hospitals with and without PCI capability, a well-trained catheterization laboratory team and the correct use of ancillary therapies are crucial prerequisites to provide the best reperfusion strategy to all patients who present with STEMI, and should be implemented in any case, in accordance with the scientific evidence and the current indications of international guidelines.

Conflict of interest statement The author received consulting fees or paid advisory boards by Sanofi Aventis, GSK, Pfizer, Astra Zeneca and lecture fees from speaking at the invitation of a commercial sponsor by Sanofi Aventis, BMS, GSK, Pfizer, Astra Zeneca, Simesa. The author received no grant support or equity ownership/stock options. The author declares that she has no conflict of interest related to the publication of this manuscript.

\section{References}

1. Van de Werf F, Ardissino D, Betriu A et al (2003) The Task Force on the management of acute myocardial infarction of the European Society of Cardiology. Management of acute myocardial infarction in patients presenting with ST-segment elevation. Eur Heart J 24:28-66

2. Grines C, Patel A, Zijlstra F et al (2003) Primary coronary angioplasty compares with intravenous thrombolytic therapy for acute myocardial infarction: six-month follow up and analysis of individual patient data from randomized trials. Am Heart $\mathrm{J}$ $145: 47-57$

3. Keeley EC, Boura JA, Grines CL (2003) Primary angioplasty versus intravenous thrombolytic therapy for acute myocardial infarction a quantitative review of 23 randomised trial. Lancet 362:13-20

4. Dalby M, Bouzamondo A, Lechat P et al (2003) Transfer for primary angioplasty versus immediate thrombolysisin acute myocardial infarction: a meta-analysis. Circulation 108:18091814

5. Stenestrand U, Lindback J, Wallentin L (2006) RIKS-HIA registry. Long-term outcome of primary percutaneous coronary intervention versus prehospital and inhospital thrombolysis for patients with ST-elevation myocardial infarction. JAMA 296:1749-1756

6. Boden WE, Eagle K, Granger CB (2007) Reperfusion strategies in acute ST-segment elevation myocardial infarction. JACC 50:917-929

7. Morrison LJ, Verbeek PR, McDonald AC et al (2000) Mortality and pre-hospital thrombolysis for acute myocardial infarction: a meta-analysis. JAMA 283:2686-2692

8. De Luca G, Suryapranata H, Ottervanger JP et al (2004) Time delay to treatment and mortality in primary angioplasty for acute myocardial infarction. Every minute of delay counts. Circulation 109:1123-1225

9. Hochman JS, Sleeper LA, Webb JG et al. For the Should We Emergently Revascularize Occluded Coronaries for Cardiogenic Shock (SHOCK) Investigators

10. Nallamothu BK, Bates ER (2003) Percutaneous coronary intervention versus fibrinolytic therapy in acute myocardial infarction: is timely (almost) everything? Am J Cardiol 92:824-826

11. Antman EM, Anbe DT, Armstrong PW et al (2004) ACC/AHA guidelines for the management of patients with ST-elevation myocardial infarction. A report of the America College of Cardiology/American Heart Association Task Force on practice guidelines (Committee to revise the 1999 guidelines for the management of patients with acute myocardial infarction). http://www.acc.org, http://www.americanheart.org

12. The Task Force for Percutaneous Coronary Intervention of the European Society of Cardiology (2005) Guidelines for percutaneous coronary interventions. Eur Heart J 26:804-847 